\title{
The Influence of Sex on Embryo Development and Pregnancy Outcome in Preimplantation Genetic Testing for Chromosomal Translocation
}

Yile Zhang ( $\sim$ luna020996@126.com )

Zhengzhou University First Affiliated Hospital

Hui Song

Zhengzhou University First Affiliated Hospital

Jing-di Li

Zhengzhou University First Affiliated Hospital

Zhi-qin Bu

Zhengzhou University First Affiliated Hospital

Fang Wang

Zhengzhou University First Affiliated Hospital

Wen-bin Niu

Zhengzhou University First Affiliated Hospital

Hao Shi

Zhengzhou University First Affiliated Hospital

\section{Research}

Keywords: preimplantation genetic testing, reciprocal translocation, Robertsonian translocation, embryonic formation, pregnancy outcome

Posted Date: September 7th, 2021

DOI: https://doi.org/10.21203/rs.3.rs-868456/v1

License: (c) (i) This work is licensed under a Creative Commons Attribution 4.0 International License. Read Full License 


\section{Abstract}

Background To investigate the embryonic development and clinical pregnancy outcome of reciprocal translocation carriers and Robertsonian translocation carriers with different sex in preimplantation genetic testing (PGT).

Methods A retrospective analysis of 1369 cycles of preimplantation genetic testing for structural rearrangements (PGT-SR) was performed in the Reproductive Medicine Center of the First Affiliated Hospital of Zhengzhou University from 2015 to 2019. All the patients were divided into reciprocal translocation and Robertsonian translocation according to the type of chromosomal translocation and divided into female carriers and male carriers according to the sex of the carriers. SPSS21.0 was used for data statistics and $\mathrm{P}<0.05$ indicated that the difference was statistically significant.

Results The fertilization rate of female carriers(81.5\%) with chromosomal structural abnormalities (including reciprocal translocation and Robertsonian translocation) was higher than that of male carriers(80.0\%) $(P=0.032)$, and the blastocyst formation rate of female carriers(50.0\%) was lower than that of male carriers $(54.8 \%)(P=0.016)$ in the same parental age. But there was no statistical difference in cleavage rate, high quality embryo rate, normal rate of biopsy, clinical pregnancy rate, abortion rate and live birth rate between female and male carriers. In the reciprocal translocation group, the blastocyst formation rate of male carriers (54.8\%) was higher than that of female carriers $(50.0 \%)(P=0.022)$ with the same parental age and there was no difference in pregnancy outcome. In the Robertsonian translocation group, the fertilization rate of male carriers $(75.0 \%)$ was lower than that of female carriers $(81.8 \%)$ $(P=0.005)$ and the normal rate of biopsy $(33.3 \%)$ was higher than that of female carriers $(25.0 \%)$ $(P=0.022)$ with the same parental age and there was no difference in pregnancy outcome.

Conclusions In reciprocal translocation, male carriers have a higher rate of blastocyst formation rate than female carriers. In Robertsonianian translocation, male carriers have a higher noamal rate of biopsy than female carriers. However, there was no significant difference in pregnancy outcome between male carriers and female carriers with abnormal chromosome structure.

\section{Background}

Reciprocal translocation, the most common chromosomal structural abnormality, is defined as the exchange of two non-homologous chromosomes after break [1][2]. Robertsonian translocation refers to the formation of two derivative chromosomes after two telomere chromosomes break at or near the centromere. In meiosis, reciprocal translocation carriers can produce at least 18 different types of gametes, of which only one is normal and one is balanced; While Robertsonian translocation carriers can produce 6 different types of gametes, and there are also only one normal and one balanced gametes [3]. Thus, although these two translocation carriers usually have a normal phenotype, the risk of infertility, miscarriage, and fetal malformations is greatly increased due to the possible formation of unbalanced gametes during meiosis [4][5]. 
The development of in vitro fertilization and advanced preimplantation molecular diagnostics provide couples at risk of translocation pregnancy with the opportunity to obtain healthy offspring [6]. The main purpose of preimplantation genetic testing for structural rearrangements (PGT-SR) is to distinguish embryos with balanced chromosomal translocation (and accompanying microdeletions) from embryos with truly normal chromosomes [7]. In recent decades, the use of preimplantation genetic testing (PGT) in assisted reproductive technology (ART) has increased significantly [8]. There are many factors that affect the reproductive outcome of PGT, including the abnormal chromosomes involved, the break point and the sex of the carrier, etc [9].

In this study, patients undergoing PGT-SR due to reciprocal translocation and Robertsonian translocation were grouped according to the sex and translocation type of carriers. The biopsy results, embryo development and pregnancy outcomes of them were analyzed and compared in order to provide ideas for in-depth understanding of the occurrence process of embryo chromosomal abnormalities and clinical evidence for the application of PGT in ART.

\section{Method}

Study Population

We collected the in vitro fertilization cycles assisted by PGT-SR in the Reproductive Medicine Center of the First Affiliated Hospital of Zhengzhou University from 2015 to 2019. The inclusion criteria was that only one of the couple is a translocation carrier (reciprocal translocation or Robertsonian translocation) and the number of eggs harvested $\geq 3$ [10]. Embryo transfer was performed for all cycles with normal biopsy results. All included couples were divided into two groups according to the type of chromosomal translocation: the reciprocal translocation group and the Robertsonian translocation group, and they were also divided into male carriers and female carriers according to the sex of carriers.

\section{Ovulation}

All patients were treated with short-acting and long-acting regimens for luteal phase [11], and the specific treatment and medication were selected according to the patients' own conditions. When the diameter of the dominant follicle reached about $18 \mathrm{~mm}$, human chorionic gonadotropin (HCG) was injected. And 36 hours later, ovarian puncture was performed under the guidance of vaginal ultrasound. The specific method adopted the conventional operation technology of our center [12].

\section{Embryo Culture and Biopsy}

We used blastocyst trophoblast biopsy for PGT-SR patients. Intracytoplasmic sperm injection was performed within 4 to $6 \mathrm{~h}$ after retrieval and the embryos were cultured to blastocyst stage at 5-6 days. Blastocysts reached or above the grade of 3BC were selected for biopsy (Blastocysts that did not reach biopsy grade were discarded after informed consent was signed by the patient). 3 to 5 trophoblast ectodermal cells were aspirated with biopsy needles combined with laser-assisted cleavage. Whole 
genome DNA of biopsied trophectoderm cell was amplified and SNP microarray chip detection technology or next-generation sequencing technology were used to detect aneuploidy.

Main Outcome Measures

The outcome measures reflecting embryo development are: fertilization rate, cleavage rate, high quality embryo rate, blastocyst formation rate and normal rate of biopsy. The outcome measures reflecting pregnancy outcome are: clinical pregnancy rate, abortion rate and live birth rate. In order to exclude the effect of age on the observed results, we also calculated the average age of the patients in male carriers and female carriers.

Statistical Analysis

SPSS21.0 was used for data statistics. Measurement data conforming to normal distribution were tested by two independent samples $t$ test and the results were expressed as Mean \pm standard deviation (Mean \pm $\mathrm{SD}$ ). And measurement data that did not conform to normal distribution were tested by non-parametric test and the results were expressed as Median \pm interquartile range (Median $\pm \mathrm{QR}$ ). The chi-square test was used for counting data, and the result of rate was expressed as percentage (\%). $\mathrm{P}<0.05$ indicated that the difference was statistically significant.

\section{Results}

Overall Situation

A total of 1369 PGT-SR cycles were included in this study, including 1006 reciprocal translocation carriers and 363 Robertsonian translocation carriers. The number of eggs obtained was 23976 and embryos biopsyed was 7540 . There were 681 cycles transplanted, and 688 cycles were cancelled to transplant due to the absence of normal transferable embryos. There were 472 female carriers and 534 male carriers in the reciprocal translocation group. And there were 181 female carriers and 182 male carriers in the Robertsonian translocation group. In the comparison of patients with chromosomal structural abnormalities (including reciprocal translocation and Robertsonian translocation) of different sex (Table 1), it can be seen that the fertilization rate of female carriers is higher than that of male carriers, while the blastocyst formation rate is lower than that of male carriers and the difference is statistically significant. But there was no statistical difference in cleavage rate, high quality embryo rate, normal rate of biopsy, clinical pregnancy rate, abortion rate and live birth rate between the two groups. 
Embryonic development and pregnancy outcome of patients with chromosomal abnormalities of different sexs

\begin{tabular}{|c|c|c|c|c|}
\hline Item & Female carrier & Male carrier & $Z / \chi^{2}$ value & $P$ value \\
\hline No. of egg retrieval cycles & 653 & 716 & / & / \\
\hline No. of retrieved oocytes & 11331 & 12645 & / & / \\
\hline Average female age $(y)$ & $29.0 \pm 6.0$ & $29.0 \pm 5.0$ & -0.143 & 0.886 \\
\hline Average female age (y) & $29.0 \pm 6.0$ & $29.0 \pm 5.8$ & -1.365 & 0.172 \\
\hline Fertilization rate (\%) & $81.5 \pm 22.0$ & $80.0 \pm 23$ & -2.139 & $0.032^{*}$ \\
\hline Cleavage rate $(\%)$ & $100.0 \pm 0.0$ & $100.0 \pm 0.0$ & -0.050 & 0.960 \\
\hline High quality embryo rate (\%) & $42.1 \pm 72.0$ & $50.0 \pm 74.0$ & -1.638 & 0.101 \\
\hline Blastocyst formation rate (\%) & $50.0 \pm 34.0$ & $54.8 \pm 37.0$ & -2.407 & $0.016^{*}$ \\
\hline No. of embryos biopsy & 3556 & 3984 & / & / \\
\hline Normal rate of biopsy (\%) & $21.4 \pm 40.0$ & $25.0 \pm 40.0$ & -1.353 & 0.176 \\
\hline No. of transplant cycles & 312 & 369 & / & / \\
\hline Clinical pregnancy rate (\%) & $56.4 \rrbracket 176 / 312 \rrbracket$ & $57.5 \rrbracket 212 / 369 \rrbracket$ & 0.075 & 0.784 \\
\hline rate of abortion (\%) & $7.4 \llbracket 23 / 312 \rrbracket$ & $10.0 \rrbracket 37 / 369 \rrbracket$ & 1.484 & 0.223 \\
\hline live birth rate (\%) & $49.0 \otimes 153 / 312 \rrbracket$ & $47.4 \rrbracket 175 / 369 \rrbracket$ & 0.105 & 0.746 \\
\hline
\end{tabular}

Notes: positive number/total number in brackets; * represents $\mathrm{P} \bigotimes 0.05$

Comparison of Reciprocal Translocation Group

In the reciprocal translocation group (Table 2), 534 PGD-SR cycles were detected in male carriers group, including 260 transplanted cycles and 274 cycles that were cancelled to transplant due to the absence of normal transferable embryos and the mean age of these male carriers was $29.0 \pm 5.0$ years and ranged from 22 to 49 years; 472 PGD-SR cycles were detected in female carriers group, including 216 transplanted cycles and 256 cycles that were cancelled to transplant due to the absence of normal transferable embryos and the mean age of these female carriers was $29.0 \pm 6.0$ years and ranged from 20 to 44 years. The blastocyst formation rate of male carriers was $54.8 \%$, which was higher than that of female carriers $(50.0 \%)$ and the difference was statistically significant. There was no statistical difference between the two in fertilization rate, cleavage rate, high quality embryo rate, normal rate of biopsy, clinical pregnancy rate, abortion rate and live birth rate. 
Table 2

Embryonic development and pregnancy outcome of reciprocal translocation carriers

\begin{tabular}{|c|c|c|c|c|}
\hline Item & Female carrier & Male carrier & $\mathrm{Z} / \chi^{2}$ value & $P$ value \\
\hline No. of egg retrieval cycles & 472 & 534 & / & / \\
\hline No. of retrieved oocytes & 8150 & 9537 & / & / \\
\hline Average female age (y) & $29.0 \pm 6.0$ & $28.0 \pm 5.0$ & -0.748 & 0.454 \\
\hline Average female age (y) & $29.0 \pm 6.0$ & $29.0 \pm 5.0$ & -1.554 & 0.120 \\
\hline Fertilization rate (\%) & $81.4 \pm 22.0$ & $81.1 \pm 22.0$ & -0.829 & 0.407 \\
\hline Cleavage rate $(\%)$ & $100.0 \pm 0.0$ & $100.0 \pm 0.0$ & -0.009 & 0.993 \\
\hline High quality embryo rate (\%) & $41.9 \pm 71.0$ & $50.0 \pm 75.0$ & -1.161 & 0.246 \\
\hline Blastocyst formation rate (\%) & $50.0 \pm 33.0$ & $54.8 \pm 34.0$ & -2.285 & $0.022^{\star}$ \\
\hline No. of embryos biopsy & 2505 & 3094 & / & / \\
\hline Normal rate of biopsy (\%) & $20.0 \pm 33.0$ & $20.0 \pm 38.0$ & -0.363 & 0.717 \\
\hline No. of transplant cycles & 216 & 260 & I & / \\
\hline Clinical pregnancy rate (\%) & $57.9 \otimes 125 / 216 \rrbracket$ & $57.3 \otimes 149 / 260 \rrbracket$ & 0.015 & 0.902 \\
\hline rate of abortion (\%) & $8.8 \rrbracket 19 / 216 \rrbracket$ & $10.8 \otimes 28 / 260 \rrbracket$ & 0.516 & 0.473 \\
\hline live birth rate (\%) & 49.1ه106/216》 & $46.5 \otimes 121 / 260 \rrbracket$ & 0.286 & 0.593 \\
\hline
\end{tabular}

Notes: positive number/total number in brackets; * represents $\mathrm{P} \bowtie 0.05$

Comparison of Robertsonian Translocation Group

In the Robertsonian translocation group (Table 3), 182 PGD-SR cycles were detected in male carriers group, including 109 transplanted cycles and 73 cycles that were cancelled to transplant due to the absence of normal transferable embryos and the mean age of these male carriers was $29.0 \pm 5.0$ years and ranged from 22 to 48 years; 181 PGD-SR cycles were detected in female carriers group, including 96 transplanted cycles and 85 cycles that were cancelled to transplant due to the absence of normal transferable embryos and the mean age of these female carriers was $29.0 \pm 4.0$ years and ranged from 20 to 41 years. The fertilization rate of male carriers was $75.0 \%$, which was lower than that of female carriers $(81.8 \%)$ but the normal rate of biopsy (33.3\%) was higher than that of female carriers $(25.0 \%)$. The differences were both statistically significant. There was no statistical difference between the two in cleavage rate, high quality embryo rate, blastocyst formation rate, clinical pregnancy rate, abortion rate and live birth rate. 
Table 3

Embryonic development and pregnancy outcome of Robertsonian translocation carriers

\begin{tabular}{|c|c|c|c|c|}
\hline Item & Female carrier & Male carrier & $Z / \chi^{2}$ value & $P$ value \\
\hline No. of egg retrieval cycles & 181 & 182 & / & / \\
\hline No. of retrieved oocytes & 3181 & 3108 & / & / \\
\hline Average female age (y) & $29.0 \pm 4.0$ & $29.0 \pm 6.0$ & -1.344 & 0.179 \\
\hline Average female age (y) & $30.0 \pm 6.0$ & $29.0 \pm 5.0$ & -0.038 & 0.970 \\
\hline Fertilization rate $(\%)$ & $81.8 \pm 22.0$ & $75.0 \pm 26.0$ & -2.816 & $0.005^{\star}$ \\
\hline Cleavage rate $(\%)$ & $100.0 \pm 0.0$ & $100.0 \pm 0.0$ & -0.068 & 0.946 \\
\hline High quality embryo rate (\%) & $42.9 \pm 78.0$ & $48.7 \pm 70.0$ & -1.314 & 0.189 \\
\hline Blastocyst formation rate (\%) & $50.0 \pm 43.0$ & $55.1 \pm 42.0$ & -0.912 & 0.362 \\
\hline No. of embryos biopsy & 1051 & 935 & / & / \\
\hline Normal rate of biopsy (\%) & $25.0 \pm 45.0$ & $33.3 \pm 39$ & -2.291 & $0.022^{*}$ \\
\hline No. of transplant cycles & 96 & 109 & / & / \\
\hline Clinical pregnancy rate (\%) & $53.1 \otimes 51 / 96 \rrbracket$ & $57.8 \varangle 63 / 109 \rrbracket$ & 0.452 & 0.502 \\
\hline rate of abortion (\%) & $4.2 \varangle 4 / 96 \rrbracket$ & $8.3 \otimes 9 / 109 \rrbracket$ & 1.438 & 0.230 \\
\hline live birth rate $(\%)$ & 49.0ه47/96》 & $49.5 \llbracket 54 / 109 \rrbracket$ & 0.054 & 0.816 \\
\hline
\end{tabular}

Notes: positive number/total number in brackets; * represents $\mathrm{P} \bowtie 0.05$

\section{Discussion}

The main purpose of this study was to compare the embryo development and pregnancy outcome in different sex in chromosomal translocation carriers (including reciprocal translocation carriers and Robertsonian translocation carriers) undergoing PGT-SR. We found by comparison that the fertilization rate of female carriers was higher than that of male carriers but the blastocyst formation rate was lower than that of male carriers. This seems to indicate that male genetic material plays an important role in the process of fertilization, and that the normal development of an embryo may be more dependent on female genetic material. Embryo chromosomes are derived from both parents. During meiosis, two translocation chromosomes and two homologous normal chromosomes form tetravalent chromosomes and separate at anaphase. Normal or balanced gametes are produced by means of alternate separation, and gametes produced by other patterns of separation have unbalanced karyotypes [13][14]. Studies have suggested that the difference in carrier sex affects the separation pattern of meiosis of tetravalent genes [14][15]. There are many factors that affect the separation pattern of meiotic chromosome, including carrier sex, maternal age, translocation chromosome type and translocation segment length, etc 
[16][17]. Therefore, we divided patients in reciprocal translocation group and Robertsonian translocation group respectively into male carriers and female carriers, and compared the influence of carrier sex on embryo development and pregnancy outcome in the same type of translocation with no difference in maternal age. In the reciprocal translocation group, the blastocyst formation rate in female carriers was lower than that in male carriers, but biopsy results and pregnancy outcome were not statistically different between the two. Female gametes are more prone to meiosis chromosome errors than male gametes [18]. This was not reflected in the biopsy results of the reciprocal translocation carriers in this study but in the blastocyst formation rate, indicating that some embryos that did not develop properly due to chromosomal abnormalities have been eliminated during embryonic development. In the Robertsonian translocation group, the fertilization rate of female carriers was higher than that of male carriers but the normal rate of biopsy was lower than that of male carriers, and the pregnancy outcome was still not statistically different. This result is comparable to the research of Li Xinyuan et al [19]. According to Munne, the meiotic segregation pattern of female carriers of Robertsonian translocation is different from that of male carriers, and the rate of producing unbalanced gametes is higher than that of male carriers [20], which is consistent with our results. However, most studies have shown that regardless of the sex of the Robertsonian translocation carrier, alternate separation is the main mode of embryo separation [21] [22]. In addition, many studies at home and abroad have shown that there is no difference in pregnancy outcome of PGT-SR between male and female carriers of chromosomal translocation [19][23], and our research have also come to the same conclusion.

At present, PGT has become a basic and important choice of assisted reproductive technology for patients with chromosomal translocation. With the continuous development and improvement of technology, more and more chromosome detection methods are applied in PGT. Fluorescence in situ hybridization (FISH), previously widely used, can only assess a discrete number of chromosomes, usually 9-12 at a time [24][25]. However, data from the evaluation of 23 pairs of chromosomes of embryonic DNA have shown that aneuploidy is relatively evenly distributed in all 23 chromosomes [26]. Therefore, FISH technology is at a significant disadvantage in the identification of aneuploidy. In this study, SNP or NGS methods were used in all cycles, which are currently more extensive method of diagnosing embryonic chromosomal abnormalities [27] and all 23 pairs of chromosomes can be comprehensively screened. It has obvious advantages compared with FISH technology, which can only detect a limited number of chromosomes [28][29][30]. In addition, compared with previous biopsy at cleavage stage, blastocyst trophoblast cell biopsy can not only control the damage of biopsy to embryos, but also ensure the accuracy of sequencing results [31].

A successful clinical pregnancy depends on many factors, such as maternal age, cause of infertility, infertility duration, endometrial receptivity, etc but only if there is a normally developing transplantable embryo. Based on a large number of PGT-SR cycles, we did a retrospective analysis and explored the effects of carriers of different sex on embryonic development and clinical pregnancy outcomes in reciprocal translocation and Robertsonian translocation, providing ideas for in-depth understanding with the process of embryonic chromosomal abnormalities. At the same time, it also provides clinical evidence for the application of PGT assisted fertility treatment. However, our study also had several limitations due 
to its retrospective design and a single medical center, and there is an inevitable bias. In addition, this research is only a preliminary discussion and the conclusions needed to be interpreted carefully.

\section{Conclusions}

In reciprocal translocation, male carriers have a higher rate of blastocyst formation rate than female carriers. In Robertsonianian translocation, male carriers have a higher noamal rate of biopsy than female carriers. However, there was no significant difference in pregnancy outcome between male carriers and female carriers with abnormal chromosome structure.

\section{Declarations}

Ethics approval and consent to participate: Not applicable

Consent for publication: Not applicable

Availability of data and materials: The data that support the findings of this study are available from Reproductive Medicine Center of the First Affiliated Hospital of Zhengzhou University but restrictions apply to the availability of these data, which were used under license for the current study, and so are not publicly available. Data are however available from the authors upon reasonable request and with permission of Reproductive Medicine Center of the First Affiliated Hospital of Zhengzhou University.

Competing interests: The authors declare that they have no competing interests.

Funding: National Natural Science Foundation of China囚3197060128\provided financial support for data collection, statistical analysis and manuscript writing.

Authors' contributions: ZYL provided research design ideas. SH completed data statistics and analysis, and was a major contributor in writing the manuscript. LJD, BZQ and WF was responsible for data collection. NWB and SH reviewed and revised the article. All authors read and approved the final manuscript.

Acknowledgements: We would like to acknowledge the professional manuscript services of American Journal Experts.

\section{References}

1. K.P Xu, H.M Yuan, I Cholst, P Chung, O Davis, I Kligman, et al. Preimplantation Genetic Diagnosis for reciprocal Chromosome Translocation. Fertil Steril. 2000; doi:10.1016/S0015-0282(00)01221-8.

2. Morin SJ, Eccles J, Iturriaga A, Zimmerman RS. Translocations, inversions and other chromosome rearrangements. Fertil Steril. 2017; doi: 10.1016/j.fertnstert.2016.10.013. 
3. Xie YX, Xu YW, Wang J, Miao BZ, Yan H, Ding CH, et al. Preliminary analysis of numerical chromosome abnormalities in reciprocal and Robertsonian translocation preimplantation genetic diagnosis cases with 24-chromosomal analysis with an aCGH/SNP microarray. Journal of assisted reproduction and genetics. 2018; doi:10.1007/s10815-017-1045-9.

4. Zhang L, Wei DM, Zhu YT, Jiang WJ, Xia MD, Li J, et al. Interaction of acrocentric chromosome involved in translocation and sex of the carrier influences the proportion of alternate segregation in autosomal reciprocal translocations. Human reproduction (Oxford, England). 2019; doi:10.1093/humrep/dey367.

5. Zhang L, Jiang WJ, Zhu YT, Chen H, Yan JH, Chen ZJ. Effects of a carrier's sex and age on the segregation patterns of the trivalent of Robertsonian translocations. J Assist Reprod Genet. 2019; doi:10.1007/s10815-019-01534-6.

6. Munné S, Fischer J, Warner A, Chen S, Zouves C, Cohen J. Preimplantation genetic diagnosis significantly reduces pregnancy loss in infertile couples: a multicenter study. Fertil Steril. 2006; doi: 10.1016/j.fertnstert.2005.10.014.

7. Treff NR, Tao X, Schillings WJ, Bergh PA, Scott RT, Levy B. Use of single nucleotide polymorphism microarrays to distinguish between reciprocal and normal chromosomes in embryos from a translocation carrier. Fertil Steril. 2011; doi: 10.1016/j.fertnstert.2011.04.038.

8. Cimadomo D, Capalbo A, Ubaldi FM, Scarica C, Palagiano A, Canipari R, et al. The Impact of Biopsy on Human Embryo Developmental Potential during Preimplantation Genetic Diagnosis. BioMed Research International. 2016; doi:10.1155/2016/7193075.

9. Rius M, Obradors A, Daina G, Ramos L, Pujol A, Martínez-Passarell O, et al. Detection of unreciprocal chromosome segregations in preimplantation genetic diagnosis of translocations by short comparative genomic hibridization. Fertil Steril. 2011; doi:10.1016/j.fertnstert.2011.04.052.

10. Chapuis A, Gala A, Ferrières-Hoa A, Mullet T, Bringer-Deutsch S, Vintejoux E, et al. Sperm quality and paternal age: effect on blastocyst formation and pregnancy rates. Basic Clin Androl. 2017; doi:10.1186/s12610-016-0045-4.

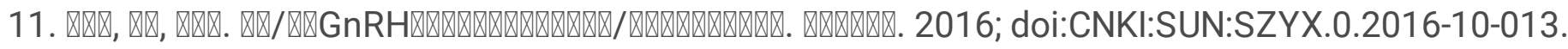

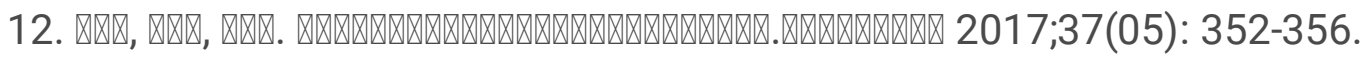

13. Scriven PN, Handyside AH, Ogilvie CM. Chromosome translocations: segregation modes and strategies for preimplantation enetic diagnosis. Prenat Diagn. 1998; doi:10.1002/(SICl)10970223(199812)18:13<1437::AID-PD497>3.0.C0;2-P.

14. Zhang L, Wei DM, Zhu YT, Jiang WJ, Xia MD, Li J, et al. Interaction of acrocentric chromosome involved in translocation and sex of the carrier influences the proportion of alternate segregation in autosomal reciprocal translocations. Hum Reprod. 2019; doi: 10.1093/humrep/dey367.

15. Ko DS, Cho JW, Park SY, Kim JY, MK, IO, et al. Clinical outcomes of preimplantation genetic diagnosis (PGD) and analysis of meiotic segregation modes in reciprocal translocation carriers. Am J Med Genet A. 2010; doi: 10.1002/ajmg.a.33368. 
16. Zhang S, Lei CX, Wu JP, Sun HY, Zhou J, Zhu SJ, et al. Analysis of segregation patterns of quadrivalent structures and the effect on genome stability during meiosis in reciprocal translocation carriers. Human reproduction (Oxford, England). 2018; doi:10.1093/humrep/dey036.

17. Ye YH, Qian YL, Xu CM, Jin F, et al. Meiotic segregation analysis of embryos from reciprocal translocation carriers in PGD cycles. Reproductive biomedicine online. 2012; doi:10.1016/j.rbmo.2011.08.012.

18. Elpida F, Santiago M, Dagan W. The cytogenetic constitution of human blastocysts: insights from comprehensive chromosome screening strategies. Human reproduction update. 2019; doi:10.1093/humupd/dmy036.

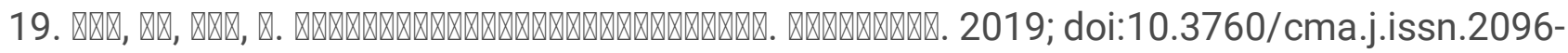
2916.2019.08.002.

20. Munne S, Escudero T, Sandalinas M, Sable D, Cohen J, et al. Gamete segregation in female carriers of robertsonian translocations. Cytogenet Cell Genet. 2000;90(3-4).

21. Bint SM, Ogilvie CM, Flinter FA, Khalaf Y, Scriven PN. Meiotic segregation of Robertsonian translocations ascertained in cleavage-stage embryos-implications for preimplantation genetic diagnosis. Human reproduction (Oxford, England). 2011; doi:10.1093/humrep/der080.

22. Scriven PN, Flinter FA, Braude PR, Ogilvie CM. Robertsonian translocations-reproductive risks and indications for preimplantation genetic diagnosis. Hum Reprod. 2001;16: 2267-73.

23. Keymolen K, Staessen C, Verpoest W, Liebaers I, Bonduelle M. Preimplantation genetic diagnosis in female and male carriers of reciprocal translocations: clinical outcome until delivery of 312 cycles. Eur J Hum Genet. 2012; doi: 10.1038/ejhg.2011.208.

24. Jobanputra V , Sobrino A, Kinney A, Kline J, Warburton D. Multiplex interphase FISH as a screen for common aneuploidies in spontaneous abortions. Hum Reprod (Oxford, England). 2002;17(5).

25. Northrop LE, Treff NR, Levy B, Scott RT. SNP microarray-based 24 chromosome aneuploidy screening demonstrates that cleavage-stage FISH poorly predicts aneuploidy in embryos that develop to morphologically normal blastocysts. Mol Hum Reprod. 2010; doi:10.1093/molehr/gaq037.

26. Brezina PR, Tobler K, Benner AT, Du L, Xu X, Kearns WG. All 23 chromosomes have significant levels of aneuploidy in recurrent pregnancy loss couples. Fertil Steril. 2012; doi: 10.1016/j.fertnstert.2012.01.014.

27. Xu JJ, Fang R, Chen L, Chen DZ, Xiao JP, Yang WM, et al. Noninvasive chromosome screening of human embryos by genome sequencing of embryo culture medium for in vitro fertilization. Proc Natl Acad Sci U S A. 2016; doi: 10.1073/pnas.1613294113.

28. Niu WB, Wang LL, Xu JW, Ying L, Shi H, Li G, et al. Improved clinical outcomes of preimplantation genetic testing for aneuploidy using MALBAC-NGS compared with MDA-SNP array. BMC Pregnancy Childbirth. 2020; doi: 10.1186/s12884-020-03082-9.

29. Coco R. Reprogenetics: preimplantational genetics diagnosis. Genetics and Molecular Biology 2014;37suppl 1. 
30. Chen HF, Chen SU, Ma GC, Hsieh ST, Tsai HD, Yang YS, et al. Preimplantation genetic diagnosis and screening: Current status and future challenges. Journal of the Formosan Medical Association. 2018; doi:10.1016/j.jfma.2017.08.006.

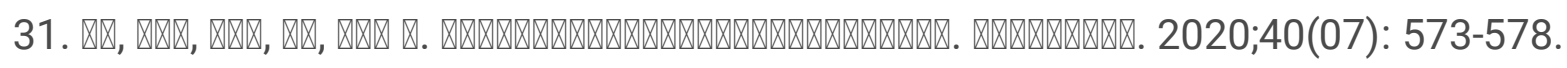

